\title{
A 10-year Study of Vertebral Osteomyelitis in Hamadan, West of Iran
}

\author{
Mojtaba Hedayat Yaghobi ${ }^{1,2}$ iD , Shahab Razipour ${ }^{3}$ (D) Mohammadmahdi Sabahi* $^{3 *}$
}

1. Dept. of Infectious Disease, Hamadan University of Medical Sciences, Hamadan, Iran

2. Dept. of infectious disease, Alborz University of Medical Sciences, Karaj, Iran.

3. Student Research Committee, Hamadan University of Medical Sciences, Hamadan, Iran

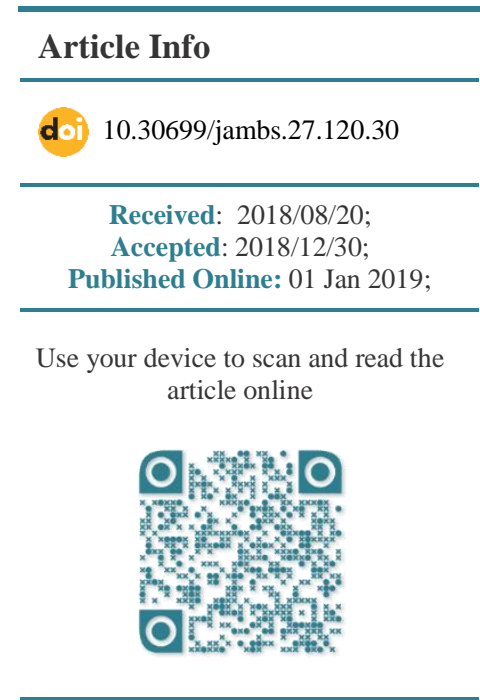

Corresponding Information: Mohammadmahdi Sabahi, Student Research Committee,Faculty of Medicine, Hamadan University of Medical Sciences, Hamadan, Iran

Email: mmsabahi1996@gmail.com

\section{ABSTRACT}

Background \& Objective: Epidemiology and predisposing factors of spondylodiscitis or vertebral osteomyelitis are different in various populations. This study was conducted to delineate the epidemiological and microbiological status of vertebral osteomyelitis in Hamadan, Iran.

Materials \& Methods: In this retrospective study, all patients with definite diagnosis of spondylodiscitis (changing of intervertebral disc and adjacent vertebral MRI signal) hospitalized in Besat and Farshchian Hospitals of Hamadan between 2006 and 2015 (during 10 years) were enrolled by convenience sampling. Data on age, gender, underlying disease, constitutional symptoms, place of acquiring infection, leukocytosis, erythrocyte sedimentation rate (ESR), C-reactive protein (CRP), surgical intervention, vertebral biopsy culture, anemia, abscess, place of vertebral involvement, positive brucellosis test, and blood culture results were obtained from the patients' medical files and recorded in a questionnaire.

Results: A total of 71 patients with spondylodiscitis (mean age: 49.56 years) were enrolled. Brucella (n: 27, 38\%) was the leading cause of the disease followed by tuberculosis (n: 11, 15.5\%). Although 34 patients had positive serologic test for Brucella, other agents were causes of the disease according to course of treatment and vertebral biopsy in 7 of them. In 21 cases, the cause of the disease was unknown. The most common place of involvement was lumbosacral region (78.9\%).

Conclusion: Unlike Infectious Diseases Society of America (IDSA) guideline that do not recommend to perform age-guided aspiration biopsy in suspected cases of spondylodiscitis when Brucella is endemic and whereby people have strong positive serology, our results demonstrated that, even in case of positive Brucella test, other factors are likely to contribute to acquiring spondylodiscitis, and vertebral biopsy is recommended for definite diagnosis. Early diagnosis is necessary to select appropriate antibiotic and treat spondylodiscitis early.

Keywords: Spondylodiscitis, Vertebral osteomyelitis (VO), Hospital acquired (HA), Community acquired (CA), PCR, Brucellosis, Tuberculosis

\section{Introduction}

Spondylodiscitis [vertebral osteomyelitis (VO)] refers to infection of the intervertebral disc or secondary osteomyelitis of adjacent vertebrae by an infectious agent (1).

Spondylodiscitis is a rare disease that accounts for $2-4 \%$ of bone and joint infections, but its incidence and diagnosis are increasing (2) due to increased elderly population and patients with chronic debilitating and immunosuppressive diseases, frequent use of invasive techniques and spinal surgery, increased prevalence of intravenous drug abuse, and improvement of diagnostic equipment especially greater availability of magnetic resonance imaging (MRI) (3). This disease is predominant in male adults, with a male/female ratio of 1.5 . The disease occurs mainly in the fifth or sixth decade of life, although people at all ages may be affected (4). The annual incidence of $\mathrm{VO}$ is 0.4-2.4 per 100,000 populations (5). VO can be hospital-acquired (HA) or community-acquired (CA) (6).
VO is a main clinical issue and a common cause of spinal surgery and long-term antibiotic treatment, and often, results in functional squeals, all of which lead to considerably increased health care costs (3). Simultaneously intervertebral disc infection and the adjacent vertebrae in majority of cases take place with hematogenous seeding (7).

VO can also occur after surgery and secondary to trauma and percutaneous procedures. Spontaneous infective spondylodiscitis affects an approximately equal number of elderly men with and without medical comorbidities (8).

Some comorbidities are known to increase the risk of pyogenic VO including diabetes, cirrhosis, immunesuppression, alcoholism, intravenous drug use, malignancy, and renal failure $(3,9)$. 
Direct spread of infection in different regions can exacerbate spondylodiscitis that leads to paravertebral (paraspinal), epidural, or psoas abscesses (10).

Pain, fever, advancement of neurological deficit and rather kyphotic deformity might be a clinical presentation of spondylodiscitis. Diagnosis in initial stages might be difficult and require imaging, laboratory and clinical evaluation (11). Overall, thoracolumbar spine, cervical spine, lumbosacral spine, lumbar spine, and thoracic spine are the mostly involved regions in spondylodiscitis (12).

The most important causative microorganisms of spondylodiscitis are Staphylococcus aureus, coagulasenegative staphylococci, Candida albicans, Brucella, and Mycobacterium tuberculosis (1, 13). In certain geographical regions, Brucella spondylodiscitis, which is a special type of spondylodiscitis, is the most common type of the disease (13).

Spontaneous spondylodiscitis features and prevalence are unexplored in the Middle East. Pyogenic infections are becoming more and more dominant over tuberculosis and Brucellosis (8). Brucella and M. tuberculosis remain endemic in Iran and many of neighboring countries, and are considered main potential causes of spondylodiscitis (7).

Since etiologic agents of spondylodiscitis are widely diverse, microbiologic culture or other rapid identification tests are used for definitive diagnosis of the causative agent. Exact identification of the agent is necessary for efficient treatment, which improves the outcome of the disease in most patients. Antibiotic treatment remains the gold standard for treating spondylodiscitis (14).

There is insufficient knowledge about clinical characteristic, entire risk factors, method of diagnosis and suitable treatment for adults with spondylodiscitis (15).

As there is no distinct signs or symptoms, diagnosis of spondylodiscitis is quite problematic. Also, other probability such as infrequency of the disease which usually lead to hold up in diagnosis after the primary appearance of symptoms and the great commonness of low back pain in the general population should be regarded (16).

Long-term mortality has increased in patients with spondylodiscitis mainly due to comorbidities especially substance abuse (17). As a result, physicians often cannot make early and proper diagnosis because of inadequate experience.

With regards to inadequate data on the epidemiology of this disease in the region, this study was conducted to investigate clinical presentations, potential risk factors, and the epidemiology of the microorganisms in patients with spondylodiscitis to plan for early diagnosis, control, and management of this disease.

\section{Materials and Methods}

In this retrospective study, all patients with definite diagnosis of spondylodiscitis hospitalized in Besat and
Farshchian Hospitals of Hamadan between 2006 and 2015 (during 10 years) were enrolled by convenience sampling. For this purpose, the medical files of hospitalized patients with definite diagnosis of spondylodiscitis were studied. Diagnosis of the spondylodiscitis was performed based on the change of the intervertebral disc and adjacent vertebral signals in MRI images. The medical files that did not fulfill inclusion criteria were excluded from the study.

The protocol of the study was approved at the Ethics Committees of the hospitals. Data on age, gender, underlying disease, constitutional symptoms, place of acquiring infection, leukocytosis, erythrocyte sedimentation rate (ESR), C-reactive protein (CRP), surgical intervention, vertebral biopsy culture, anemia, abscess, place of vertebral involvement, positive Brucellosis test, and blood culture result were drawn from the patients. All the above procedures were done in Besat and Farshchian Hospitals and Farzan laboratory of Hamadan. The data drawn from medical files were recorded in a questionnaire.

Diagnosis of Brucellosis was made by serologic tests such as Wright, 2ME, and Coombs-Wright, and standard agglutination test (SAT) results of $\geq 1: 160$ were considered to indicate diagnosis of Brucellosis (1).

Diagnosis of Tuberculosis was made by positive polymerase chain reaction test for tuberculosis (TB PCR).

Data analysis was conducted by Chi-square test, ANOVA, and descriptive statistics using SPSS 16 (SPSS Inc., Chicago IL., USA).

\section{Results}

A total of 71 patients with spondylodiscitis [63.4\% males, 36.6\% females, mean age: 49.56 (range: 17-84) years)] were enrolled. Sixty-one patients $(85.9 \%)$ had CA spondylodiscitis and $10 \quad(14.1 \%)$ had HA spondylodiscitis. Of 61 patients with CA spondylodiscitis, $41(67.2 \%)$ were male and of 10 patients with HA spondylodiscitis, six $(60 \%)$ were female.

Regarding etiology, 34 patients had positive serologic Brucella test; in 27 (38\%) of these patients, Brucella had been treated according to the disease course and vertebral biopsy, and in seven patients, another causes were the reason for spondylodiscitis despite positive serologic Brucella test. In addition to Brucella, tuberculosis (n: 11, 15.5\%), S. aureus (n: 4, 5.7\%), Staphylococcus epidermidis (n: 1, 1.4\%), C. albicans (n: 3, 4.2\%), and Klebsiella pneumoniae (n: 3, 4.2\%) were also isolated from vertebral biopsy (Table 1).

Of three cases with $C$. albicans, one was diagnosed by BACTEC culture medium and two were diagnosed by PCR. In one patient, infection occurred due to both $C$. albicans and $S$. aureus. In 21 patients, vertebral biopsy and culture as well as serologic tests did not show any microorganism, and the patients were undergone antibiotic treatment and responded to the treatment. 
Table 1. Microorganisms distribution

\begin{tabular}{|cc|}
\hline Frequency $(\%)$ & Microorganism \\
\hline $11(15.5 \%)$ & Mycobacterium tuberculosis \\
\hline $27(38 \%)$ & Brucellaspp \\
$3(4.2 \%)$ & Candida \\
$4(5.7 \%)$ & Staphylococcus aureus \\
$1(1.4 \%)$ & Staphylococcus epidermidis \\
$21(29.6 \%)$ & Pyogenic \\
$3(4.2 \%)$ & Klebsiella \\
$1(1.4 \%)$ & Staph \& Candida \\
$71(100 \%)$ & Total \\
\hline
\end{tabular}

Age was significantly associated with the cause of spondylodiscitis $(P=0.031)$ such that Brucella was the most frequent cause of the infection in the young patients and tuberculosis in older patients.

Overall, the most common place of involvement was lumbosacral spine $(78.9 \%)$ followed by thoracic spine (9.9\%), cervical spine (7\%), and thoracolumbar spine (4.2\%). In patients with Brucellar spondylodiscitis, lumbosacral spine $(74.1 \%)$ was the most frequently involved region followed by thoracic spine (14.8\%), cervical spine $(7.4 \%)$, and thoracolumbar spine $(3.7 \%)$. Regarding tuberculosis, lumbosacral spine $(81.8 \%)$ was the most frequently involved region (Table 2).

In this study, abscess was observed in $60.5 \%$ of the patients, paraspinal abscess in $33.8 \%$ (n: 24) of the patients, epidural abscess in $19.7 \%$ (n: 14), and psoas abscess in 7\% (n: 5). Paraspinal abscess was observed in $63.6 \%$ of cases of spondylodiscitis due to tuberculosis and $25.9 \%$ of cases of spondylodiscitis due to C. albicans, and epidural and psoas abscesses were more common in cases of indistinctive-microorganism-induced spondylodiscitis.

In cases of spondylodiscitis due to Brucella, only one psoas abscess and no epidural abscess was observed. Regarding underlying predisposing diseases, diabetes mellitus $(11.3 \%)$ was the most common risk factor (Table 3).

Regarding clinical presentations, constitutional symptoms consisting of fever, night sweats, and weight loss were seen in $32(45.1 \%)$ patients, anemia in $66.2 \%$, leukocytosis in $21.1 \%$, positive CRP in $67.6 \%$, ESR > 100 in $11.3 \%$ of the patients, elevated ESR in $63.4 \%$, and normal ESR in $25.4 \%$.

Table 2. Vertebral involvement of patients with Spondylodiscitis

\begin{tabular}{cccccc} 
Total & $\begin{array}{c}\text { Thoracolumbar } \\
\text { tract }\end{array}$ & $\begin{array}{c}\text { Lumbosacral } \\
\text { tract }\end{array}$ & $\begin{array}{c}\text { Thoracic } \\
\text { tract }\end{array}$ & $\begin{array}{c}\text { Cervical } \\
\text { tract }\end{array}$ & $\begin{array}{c}\text { Letter name } \\
11(15.5 \%)\end{array}$ \\
$1(9.1 \%)$ & $9(81.8 \%)$ & $1(9.1 \%)$ & 0 & Mycobacterium tuberculosis \\
$27(38 \%)$ & $1(3.7 \%)$ & $20(74.1 \%)$ & $4(14.8 \%)$ & $2(7.4 \%)$ & Brucella. spp \\
$3(4.2 \%)$ & 0 & $2(66.7 \%)$ & $1(33.3 \%)$ & 0 & Candida \\
$5(7.1 \%)$ & 0 & $4(80 \%)$ & 0 & $1(20 \%)$ & Staph \\
$21(29.6 \%)$ & $1(4.8 \%)$ & $18(85.7 \%)$ & 0 & $2(9.5 \%)$ & Pyogenic \\
$3(4.2 \%)$ & 0 & $2(66.7 \%)$ & $1(33.3 \%)$ & 0 & Klebsiella \\
$1(1.4 \%)$ & 0 & $1(100 \%)$ & 0 & 0 & Staph \& Candida \\
$71(100 \%)$ & $33(4.2 \%)$ & $56(78.9 \%)$ & $77(9.9 \%)$ & $55(7 \%)$ & Total \\
\hline
\end{tabular}

Table 3. Demographic characteristics

\begin{tabular}{|c|c|c|c|c|c|}
\hline P- value & $\begin{array}{c}\text { Total } \\
(\mathbf{N}=71)\end{array}$ & $\begin{array}{c}\text { Other } \\
\text { Microorganisms } \\
(\mathbf{N}=\mathbf{3 3})\end{array}$ & $\begin{array}{l}\text { M. tuberculosis } \\
(\mathrm{N}=11)\end{array}$ & $\begin{array}{c}\text { Brucella } \\
(\mathrm{N}=27)\end{array}$ & \\
\hline 0.832 & $63.4 \% / 36.6 \%$ & $22 / 11$ & $7 / 4$ & $16 / 11$ & Male/female \\
\hline $0.031 *$ & $49.56 \pm 16.38$ & $50.69 \pm 15.68$ & $59.5 \pm 13.65$ & $\begin{array}{c}44.11 \pm 16.5 \\
3\end{array}$ & Age (years) \\
\hline 0.435 & $32(45.1 \%)$ & $16(48.5 \%)$ & $3(27.3)$ & $13(48.1 \%)$ & $\begin{array}{l}\text { Constitutional } \\
\text { Symptoms }\end{array}$ \\
\hline 0.615 & $47(66.2 \%)$ & $23(69.7 \%)$ & $8(72.7 \%)$ & $16(59.2 \%)$ & Anemia \\
\hline $0.014^{*}$ & $15(21.1 \%)$ & $12(36.3 \%)$ & $1(9.1 \%)$ & $2(7.4 \%)$ & Leukocytosis \\
\hline \multirow{2}{*}{0.066} & $61(85.9 \%)$ & $25(75.7 \%)$ & $10(90.9 \%$ & 26(96.3) & $\mathrm{CA}$ \\
\hline & $10(14.1 \%)$ & $8(24.3 \%)$ & $1(9.1 \%)$ & $1(3.7 \%)$ & HA \\
\hline 0.380 & $43(60.5 \%)$ & $19(57.5 \%)$ & $11(100 \%)$ & $13(48.1 \%)$ & Abscess \\
\hline \multirow{3}{*}{0.310} & $8(11.3 \%)$ & $2(6 \%)$ & $1(9.1 \%)$ & $5(18.5 \%)$ & Diabetes \\
\hline & $2(1.4 \%)$ & $1(3 \%)$ & $1(9.1 \%)$ & $0(0 \%)$ & $\begin{array}{l}\text { chronic renal } \\
\text { failure (CRF) }\end{array}$ \\
\hline & $2(2.8 \%)$ & $2(6 \%)$ & $(0 \%)$ & $0(0 \%)$ & Cardiovascular \\
\hline
\end{tabular}

\section{Discussion}

A total of 71 patients with spondylodiscitis (mean age: 49.56 years) were enrolled. Brucella (n: 27, 38\%) was the leading cause of the disease followed by tuberculosis (n: 11, 15.5\%). Although 34 patients had 
positive serologic test for Brucella, other agents were causes of the disease according to course of treatment and vertebral biopsy in 7 of them. In 21 cases, the cause of the disease was unknown. The most common place of involvement was lumbosacral region (78.9\%). Unlike IDSA guideline that do not recommend to perform image-guided aspiration biopsy in suspected cases of spondylodiscitis when Brucella is endemic and whereby people have strong positive serology, our study demonstrated that mere positive serologic Brucella test did not represent vertebral involvement by this microorganism, as in seven out of 34 cases with positive serologic test, treatment with Brucella drug regimen did not cause improvement and relief of symptoms, and biopsy was necessary to diagnose the cause of vertebral involvement. Of these seven cases, four had spondylodiscitis due to M. tuberculosis and three due to indistinctive species $(18,19)$.

As expected, spondylodiscitis was more frequently seen in the elderly. In the current study, the mean age of the patients was $49.56 \pm 16.38$ years and patients with an age of 65 or older (14 patients) were considered the elderly. In two separate studies in Italy and Turkey, the mean age of the patients was 57.7 (6) and 52.5 (18) years, respectively, and in England, $73 \%$ of the patients were over 65 years (20).

Infectious spondylodiscitis occurs mainly in the elderly population, who are more frequently exposed to healthcare contacts. As a result, a growing number of multi-resistant bacteria and fungi are the causes of the infection (6).

In our study, Brucellar spondylodiscitis was the most frequently seen spondylodiscitis with prevalence rate of $38 \%$. According to previous studies, the prevalence rate of Brucellar spondylodiscitis is $2-58 \%$ (21). In 469 patients hospitalized due to HA Brucellosis in northern Iran, the prevalence of Brucellar spondylodiscitis was reported approximately $6.2 \%$ (22). In most previous studies, even in developed countries, S. aureus has been the most common cause of $\operatorname{VO}(\mathbf{1}, 3,6,8,23)$. Besides that, in other studies, M. tuberculosis, coagulase negative Streptococci, and Brucella spp. were reported to be the cause of the disease $(1,7,11)$.

The most frequent reasons for pyogenic infection of spine in Ahvaz (southwest of Iran), were S. aureus $(28.5 \%)$ and $M$. tuberculosis (42.9\%) (24).

In an epidemiologic study on 85 patients with spondylodiscitis, M. tuberculosis (43 patients) and $S$. aureus (15 patients) were found as the main pathogens (22). However, in the region studied in the current work, Brucella spp., followed by M. tuberculosis, was the main cause of the disease due to being endemic; in approximately $1 / 3$ of patients with pyogenic spondylodiscitis in previous studies, the infectious agent was never detected (24).

In the current study, $67.2 \%$ of patients with CA spondylodiscitis were male. Consistently, CA spondylodiscitis has been reported to be more common in men and HA spondylodiscitis is more common in women $(3,10,25)$.

In a study, spondylodiscitis was reported to be predominant in male patients only in the CA spondylodiscitis group, whereas among HA spondylodiscitis patients, there was an approximately equal number of men and women (6).

Constitutional symptoms such as fever, local back pain, night sweats, limitation of motion, weight loss, and neurologic abnormalities are common but are not significantly associated with development of the disease (24, 26-28).

Neurologic deficit symptoms are a prognostic unpleasant sign that represent an adverse outcome (6). In previous studies, back pain was the most frequently reported symptom, followed by fever $(6,26,27)$. In our study, fever, night sweats, and weight loss were seen in $45.1 \%$ of the patients; and notably, Brucella was the main cause of spondylodiscitis, which is not consistent with cited studies. The site of pain depends on the site of infection. Overall, the most common involved region was lumbosacral spine followed by thoracic spine, cervical spine, and thoracolumbar spine, which is consistent with most previous studies irrespective of the cause of spondylodiscitis (6). For example, the most common site in a study was the lumbar spine (58\%), followed by the thoracic spine $(30 \%)$ and the cervical spine (11\%) (26). In another study, the most common site of infection was the lumbar spine $(66.7 \%)$, followed by the thoracic spine (19\%), the sacroiliac joint $(9.5 \%)$, and the lumbar-thoracic spine $(4.8 \%)$. The L3-L4 site $(23.8 \%)$ was also reported to be the most frequent site of infection (24).

Spondylodiscitis patients, in previous studies, have been reported to suffer from paravertebral abscess (13\%) (28) and (26\%) (10), epidural abscess (10.2\%) (28), (17\%) (10), and (46.25\%) (18), psoas abscess (3.4\%), and radiculitis $(2.7 \%)(29)$.

In the present study, paraspinal abscess was the most frequently seen abscess $(33.8 \%)$, and $60.5 \%$ of all cases had abscess. This increased incidence rate of abscess can be attributed to the causes of spondylodiscitis in the studied population. Notably, in our study, in most cases with paraspinal abscess, Brucella and M. tuberculosis were the causes of the disease due to the endemicity of these two microorganisms in the studied region. Abscess was seen in all cases with spondylodiscitis due to M. tuberculosis.

A study reported that about $5 \%$ of patients with spinal epidural abscesses died, mainly due to sepsis, uncontrolled meningitis, and other underlying diseases (30). Therefore, due to highly frequent incidence of abscess in spondylodiscitis patients in the studied population in our study, it is essential to diagnose this disease earlier in the population of the region. Studies have also indicated that in patients with abscess, recurrence of the disease and clinical complications following relative recovery occur more frequently. In 
addition, the treatment process of patients with abscess, occasionally requires surgery (12).

Leukocytosis was significantly less frequent in patients with spondylodiscitis due to M. tuberculosis and Brucella compared to other pathogenic causes of the disease $(P=0.014)$; and leukocytosis or increased number of neutrophils is not adequately sensitive to diagnose spondylodiscitis $(20,24,31)$ and is not a specific sign of spondylodiscitis.

In contrast to leukocytosis, increased ESR and CRP levels are highly sensitive and have been reported in $98 \%$ and $100 \%$ of spondylodiscitis cases, respectively $(31,32)$, which is relatively consistent with our study as in $63.4 \%$ of our patients, ESR was higher than normal.

The mean ESR was obtained $48 \mathrm{~mm} / \mathrm{h}$ in a study conducted in Iran between 1971 and 1995 (33). In other studies, CRP and ESR levels in spondylodiscitis patients significantly increased $(\mathbf{1 0}, \mathbf{3 2})$. However, clinical diagnosis of spondylodiscitis confirms by CRP and ESR, which are convenient diagnostic and prognostic methods and are practical in the follow-up treatment. ESR that represents inflammatory state, is consistently high in patients with spinal infection (24).

The percentages of anemia in cases of spondylodiscitis due to Brucella and M. tuberculosis were $59.2 \%$ and $72.7 \%$, respectively. It has been reported that anemia is the most common hematological disorder in patients with Brucellosis, and considerably common in older and chronic disease patients (34). In the present study, 13 patients suffered from underlying diseases ( 8 diabetes, 2 chronic renal failure, and 2 cardiovascular disease).

Among VO patients in another study 24 cases $(53.3 \%)$ had one or more comorbidities (32), and in the study of Bhavan et al., diabetes and CRF were the most frequent comorbidities (23). In a study, $32.5 \%$ of population had comorbidity (18).

In a similar retrospective observational study conducted within 4 years in Oman, 62 primary spondylodiscitis cases were detected, including 10 cases of tuberculosis (17\%), one case of Brucellosis, and others suffering from pyogenic infection. $63.8 \%$ were male. Common associated illnesses were diabetes mellitus (n: 18), hypertension ( $\mathrm{n}$ : 13), and renal insufficiency (n: 6). Lumbar spine was involved in $69 \%$ of patients $(8)$.

It's vital to get blood cultures in order to asses VO. A positive result is indicative of the need for more invasive procedures. A systemic review on VO reported that out of all cases, $58 \%$ had positive blood cultures. (26).

MRI findings and microbiological methods are essential to make an appropriate diagnosis of pyogenic spondylodiscitis (24). In a study on two spondylodiscitis cases, the Brucella serologic test of both cases was negative, but one of them had positive blood culture and the other one had positive vertebral needle biopsy for Brucella (35).

Serology tests are inexpensive, but it should be noted these tests may be initially negative and then become positive with the development of Brucellosis (28).

In the study of Horasan et al. on patients with Brucellar VO, the diagnostic tool in $90 \%$ of patients was serology tests, while in the rest of patients, the microorganism involved was detected by blood culture (18).

A 9-year study demonstrated that minimally invasive surgery is effective and risk-free for patients with spontaneous pyogenic thoracic and lumbar spondylodiscitis (2)

\section{Conclusion}

This study showed the important role of Brucellosis and tuberculosis, as two endemic causes, in acquiring spondylodiscitis in the studied region. Although Brucella was the most frequent cause of spondylodiscitis, other infectious agents should be considered potential causes of this disease. In this study, anemia, elevated ESR, and positive CRP were found to be comparatively more frequent symptoms of spondylodiscitis than other clinical presentations. In regions where both tuberculosis and Brucella are endemic, even in case of positive serologic Brucella test, other agents are likely to contribute to acquiring spondylodiscitis; and vertebral biopsy is recommended for definite diagnosis of spondylodiscitis. These results are unlike IDSA guideline that do not recommend to perform age-guided aspiration biopsy in suspected cases of spondylodiscitis. Early diagnosis assists in selecting appropriate antibiotic and early treatment.

\section{Acknowledgements}

All the ethical standards of the institutional and/or national research committee and the 1964 Helsinki declaration and its further revisions or comparable ethical standards were observed in all procedures including human participants carried out in researches. Formal consent is not needed for this kind of study.

\section{Conflict of Interest}

Authors declared no conflict of interests.

\section{References}

1. Butler JS, Shelly MJ, Timlin M, Powderly WG, O'Byrne JM. Nontuberculous pyogenic spinal infection in adults: a 12year experience from a tertiary referral center. Spine (Phila Pa 1976). 2006; 31(23): 2695-700. [DOI:10.1097/01.brs .0000244662.78725.37] [PMID]

2. Viezens L, Schaefer C, Helmers R, Vettorazzi E, Schroeder M, Hansen-Algenstaedt N. Spontaneous pyogenic spondylodiscitis in the thoracic or lumbar spine: a retrospective cohort study comparing the safety and efficacy of minimally invasive and open surgery over a nine-year 
period. World Neurosurg. 2017; 102: 18-27. [DOI:10.1016/j. wneu.2017.02.129] [PMID]

3. Cottle L, Riordan T. Infectious spondylodiscitis. J Infect. 2008; 56(6): 401-12. [DOI:10.1016/j.jinf.2008.02.005] [PMID]

4. Grammatico L, Baron S, Rusch E, et al. Epidemiology of vertebral osteomyelitis (VO) in France: analysis of hospitaldischarge data 2002-2003. Epidemiol Infect. 2008; 136(5): 653-60. [DOI:10.1017/S0950268807008850] [PMID] [PMCID]

5. Premru MM, Spik VC, Furlan SL, Zupanc TL. Clinical appearance of Staphylococcus aureus spondylodiscitis and molecular characterization of the isolates. Scand J Infect Dis. 2010; 42(10): 763-6. [DOI:10.3109/00365548 .2010.492398] [PMID]

6. D'Agostino C, Scorzolini L, Massetti AP, et al. A seven-year prospective study on spondylodiscitis: epidemiological and microbiological features. Infection. 2010; 38(2): 102-7. [DOI:10.1007/s15010-009-9340-8] [PMID]

7. Skaf GS, Kanafani ZA, Araj GF, Kanj SS. Non-pyogenic infections of the spine. Int J Antimicrob Agents. 2010; 36(2): 99-105. [DOI:10.1016/j.ijantimicag.2010.03.023] [PMID]

8. Menon KV, Sorour TM. Epidemiologic and demographic attributes of primary spondylodiscitis in a middle eastern population sample. World Neurosurg. 2016; 95: 31-9. [DOI:10.1016/j.wneu.2016.07.088] [PMID]

9. Govender S. Spinal infections. J Bone Joint Surg Br. 2005; 87(11): 1454-8. [DOI:10.1302/0301-620X.87B11.16294] [PMID]

10. McHenry MC, Easley KA, Locker GA. Vertebral osteomyelitis: long-term outcome for 253 patients from 7 Cleveland-area hospitals. Clin Infect Dis. 2002; 34(10): 1342-50. [DOI:10.1086/340102] [PMID]

11. Guerado E, CervanAM. Surgical treatment of spondylodiscitis. An update. Int Orthop. 2012; 36(2): 41320. [DOI:10.1007/s00264-011-1441-1] [PMID] [PMCID]

12. Kaptan F, Gulduren HM, Sarsilmaz A, et al. Brucellarspondylodiscitis: comparison of patients with and without abscesses. Rheumatol Int. 2013; 33(4): 985-92. [DOI:10.1007/s00296-012-2491-4] [PMID]

13. Pappas G, Papadimitriou P, Akritidis N, Christou L, Tsianos EV. The new global map of human Brucellosis. Lancet Infect Dis. 2006; 6(2): 91-9. [DOI:10.1016/S1473-3099(06)70382-6]

14. Soares do, Brito J, Tirado A, Fernandes P. Surgical treated spondylodiscitis epidemiological study. Acta Med Port. 2016; 29(5): 319-25. [DOI:10.20344/amp.6549] [PMID]

15. Cebrian Parra JL, Saez-Arenillas Martin A, Urda MartinezAedo AL, SolerIvanez I, Agreda E, Lopez-Duran Stern L. Management of infectious discitis. Outcome in one hundred and eight patients in a university hospital. Int Orthop. 2012; 36(2): 239-44. [DOI:10.1007/s00264-011-1445-x] [PMID] [PMCID]

16. Glaudemans AW, Quintero AM, Signore A. PET/MRI in infectious and inflammatory diseases: will it be a useful improvement? Eur J Nucl Med Mol Imaging. 2012; 39(5): 745-9. [DOI:10.1007/s00259-012-2060-9] [PMID] [PMCID]

17. Aagaard T, Roed C, Dahl B, Obel N. Long-term prognosis and causes of death after spondylodiscitis: A Danish nationwide cohort study. Infect Dis (Lond). 2016; 48(3): 201-8. [DOI:10.3109/23744235.2015.1103897] [PMID]

18. Horasan ES, Colak M, Ersoz G, Uguz M, Kaya A. Clinical findings of vertebral osteomyelitis: Brucella spp. versus other etiologic agents. Rheumatol Int. 2012; 32(11): 3449 53. [DOI:10.1007/s00296-011-2213-3] [PMID]

19. Berbari EF, Kanj SS, Kowalski TJ, et al. Infectious Diseases Society of America (IDSA) clinical practice guidelines for the diagnosis and treatment of native vertebral osteomyelitis in adults. Clin Infect Dis. 2015; 15; 61(6): e26-46. [DOI:10.1093/cid/civ482] [PMID]

20. Hopkinson N, Stevenson J, Benjamin S. A case ascertainment study of septic discitis: clinical, microbiological and radiological features. QJM. 2001; 94(9): 465-70. [DOI:10.1093/qimed/94.9.465] [PMID]

21. Doganay M, Aygen B. Human Brucellosis: an overview. Int J Infect Dis. 2003; 1; 7(3): 173-82. [DOI:10.1016/S12019712(03)90049-X]

22. Hasanjani Roushan MR, Mohrez M, SmailnejadGangi SM, SoleimaniAmiri MJ, Hajiahmadi M. Epidemiological features and clinical manifestations in 469 adult patients with Brucellosis in Babol, Northern Iran. Epidemiol Infect. 2004; 132(6): 1109-14. [DOI:10.1017/S0950268804002833] [PMID] [PMCID]

23. Bhavan KP, Marschall J, Olsen MA, Fraser VJ, Wright NM, Warren DK. The epidemiology of hematogenous vertebral osteomyelitis: a cohort study in a tertiary care hospital. BMC Infect Dis. 2010; 10: 158. doi: 10.1186/1471-2334-10-158. [DOI:10.1186/1471-2334-10-158] [PMID] [PMCID]

24. Sheikh AF, Khosravi AD, Goodarzi H, et al. pathogen identification in suspected cases of pyogenic spondylodiscitis. Front Cell Infect Microbiol. 2017; 7: 60. [DOI:10.3389/fcimb.2017.00060] [PMID] [PMCID]

25. Solis Garcia delPozo J, Vives Soto M, Solera J. Vertebral osteomyelitis: long-term disability assessment and prognostic factors. J Infect. 2007; 54(2): 129-34. [DOI:10.1016/j.jinf.2006.01.013] [PMID]

26. Mylona E, Samarkos M, Kakalou E, Fanourgiakis P, Skoutelis A. Pyogenic vertebral osteomyelitis: a systematic review of clinical characteristics. Semin Arthritis Rheum. 2009; 39(1): 10-7. [DOI:10.1016/j.semarthrit.2008.03.002] [PMID]

27. Kaya S, Ercan S, Kaya S, et al. Spondylodiscitis: evaluation of patients in a tertiary hospital. J Infect Dev Ctries. 2014; 8(10): 1272-6. [DOI:10.3855/jidc.4522] [PMID]

28. Hu T, Wu J, Zheng C, Wu D. Brucellarspondylodiscitis with rapidly progressive spinal epidural abscess showing caudaequina syndrome. Spinal Cord Ser Cases. 2016; 2: 15030. [DOI:10.1038/scsandc.2015.30] [PMID] [PMCID]

29. Ulu-Kilic A, Karakas A, Erdem H, et al. Update on treatment options for spinal Brucellosis. Clin Microbiol Infect. 2014; 20(2): O75-82. [DOI:10.1111/1469-0691.12351] [PMID]

30. Shioya N, Ishibe Y, Kan S, et al. Sternoclavicular joint septic arthritis following paraspinal muscle abscess and septic lumbar spondylodiscitis with epidural abscess in a patient with diabetes: a case report. BMC Emerg Med. 2012; $12: 7$. [DOI:10.1186/1471-227X-12-7] [PMID] [PMCID]

31. Jensen AG, Espersen F, Skinhoj P, Frimodt-Moller N. Bacteremic Staphylococcus aureus spondylitis. Arch Intern 
Med. 1998; 158(5): 509-17. [DOI:10.1001/archinte. 158.5.509] [PMID]

32. Khan MH, Smith PN, Rao N, Donaldson WF. Serum Creactive protein levels correlate with clinical response in patients treated with antibiotics for wound infections after spinal surgery. Spine J. 2006; (3): 311-5. [DOI:10.1016/j.spinee.2005.07.006] [PMID]
33. Jabalameli M, Ameri E. Bone and joint tuberculosis, Review of the patients treated in the ShafaYahiaian Hospital, 135074. Tehran Univ Med J. 1998; 56(4): 62-6.

34. Abdi-Liae Z, Soudbakhsh A, Jafari S, Tomaj HE. Haematological manifestations of Brucellosis. Acta MedicaIranica. 2007; 45(2): 145-8.

35. Celik AD, Yulugkural Z, Kilincer C, Hamamcioglu MK, Kuloglu F, Akata F. Negative serology: could exclude the diagnosis of Brucellosis? Rheumatol Int. 2012; 32(8): 2547 9. [DOI:10.1007/s00296-010-1453-y] [PMID]

\section{How to Cite This Article:}

Hedayat yaghobi M, Razipour S, Sabahi MM. A 10-years study of vertebral osteomyelitis in Hamadan, West of Iran. J Adv Med Biomed Res. 2019; 27 (120) :30-36

\section{Download citation:}

BibTeX | RIS | EndNote | Medlars | ProCite | Reference Manager | RefWorks

\section{Send citation to:}

(8). Mendeley 2 Zotero (i)efWorks RefWorks 